\title{
A DEEP WIDE-FIELD SURVEY FROM DIGITALLY STACKED SCHMIDT PLATES
}

\author{
M.R.S. HAWKINS \\ Royal Observatory \\ Blackford Hill \\ Edinburgh EH9 $3 \mathrm{HJ}$ \\ Scotland
}

Over the last 18 years or so a large-scale monitoring programme has been undertaken with the UK $1.2 \mathrm{~m}$ Schmidt telescope in field 287 at $21 \mathrm{~h} 28 \mathrm{~m},-45 \mathrm{deg}$. Plates have been taken over all timescales from 1 hour to 17 years in red and blue passbands, and large numbers of plates have also been taken in other passbands to give deep coverage in $U, B, V, R$ and $I$. The field is calibrated with some $200 \mathrm{CCD}$ and photoelectric standards, and covers approximately 25 square degrees.

Although it was originally the intention to take these plates for the detection and monitoring of long term variables, especially quasars, a number of new projects based on this plate material are now being undertaken. To data there are some 200 deep plates of the field, including 65 IIIa-J, 65 IIIa-F, 30 IV-N and smaller numbers in other passbands including $U$ and V.

The idea of digitally stacking the plates to obtain greater depth has been an attractive possibility from the start, but only recently has improvement in computer technology made this a feasible undertaking. During the past two years a procedure for plate stacking has been developed, and deep arrays in several passbands have been obtained, comprising some $65 \mathrm{~B}, 65$ $R, 30 \mathrm{I}, 7 \mathrm{U}$ and $3 \mathrm{~V}$ band plates. The resulting limiting magnitudes are roughly $B=26, R=24$, $I=22, U=24$ and $V=22$. By stacking plates in smaller subgroups it is possible to measure proper motions and look for variability, although clearly with a somewhat reduced limiting magnitude.

The resulting database combines large area coverage with deep limiting magnitude in an unprecedented way. Many projects are planned including searches for brown dwarfs, cool white dwarfs and halo stars. The survey for variable quasars will be extended to fainter magnitudes and higher redshift. A number of large-scale structure projects are planned using deep wide area samples of galaxies and galaxy clusters. The field has already been mapped with the Molongolo Radio Telescope, and it is hoped to combine these data with surveys in other passbands including infrared and X-ray.

The stacked digitised data is held in the same format as the original COSMOS mapping mode files. This means that the COSMOS analyzer and other software can be run on it directly, or after some conversion of the photographic density to intensity. The software detects images above a threshold related to the sky background and outputs a number of parameters for each image, including position, integrated intensity and an elliptical fit to the image. There is also the option to 'deblend' nearby objects which have been measured as one image.

This new deep regime presents some difficulties which are not associated with single plate 
measures. In the 25 square degrees which were measured, some one million images were detected. This is about half what one would expect from counts on deep CCD frames. The reason for the deficit is the much larger number of overlapping images. This will reduce the effective area when compiling a sample of uncontaminated objects, and complicate such measures as two point correlation functions. A second difficulty concerns the discrimination of star-like images from galaxies. The stacking process results in an effective seeing disc somewhat larger than a single survey plate, due to the necessity to include many plates which were not taken in excellent seeing. This, combined with the fact that at such faint magnitudes the average galaxy image is significantly smaller than on a single plate, means that star/galaxy separation techniques which work well on single plates are ineffective when applied to the stacked data.

Despite these problems, there are no difficulties in calibrating the data with a CCD sequence in the normal way. Figure 1 shows the relation between COSMOS magnitudes and CCD magnitudes for the IIIa-J stack. The scatter is around $0.1 \mathrm{mag}$, slightly larger than for a good single plate, and interestingly the scatter only increases slightly with magnitude.

Astrometric measures are a fundamental part of a number of potential projects, including searches for brown dwarfs, cool white dwarfs and halo stars. In order to measure proper motions the data were stacked in groups of four or five plates taken within a year. This resulted in an increase in depth of around one magnitude over a single plate, with an error of only 20 mas/yr. Figure 2 shows a histogram of the measured proper motions for all objects with a peak at around 20 mas and a long tail of proper motion objects.

One of the first projects to be tackled with the stacked data was the search for Brown Dwarfs.

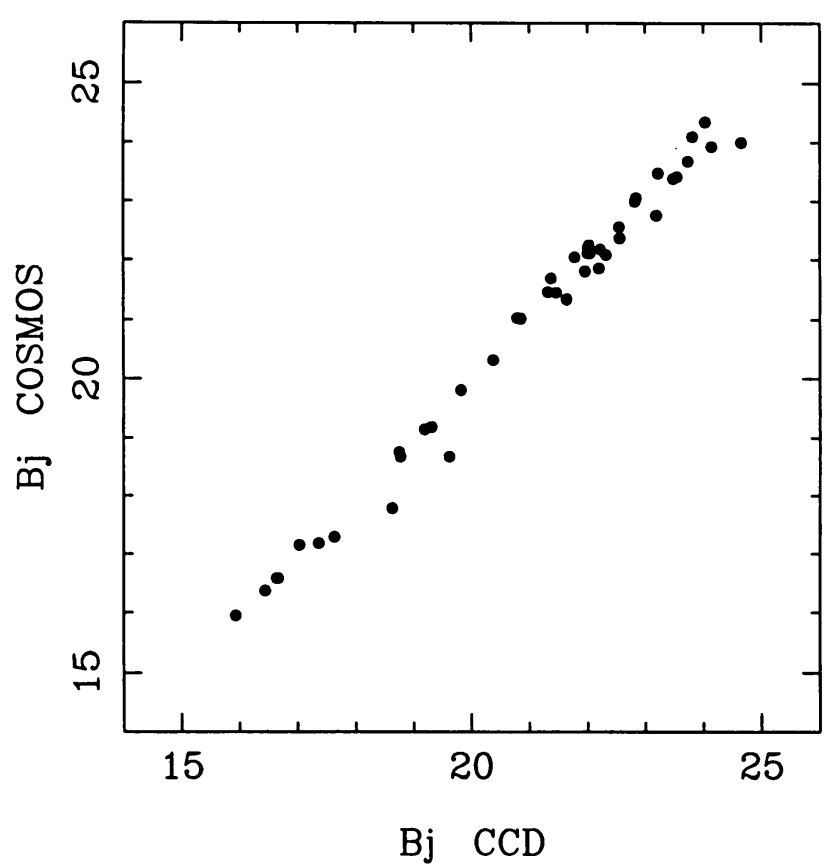

Figure 1. 


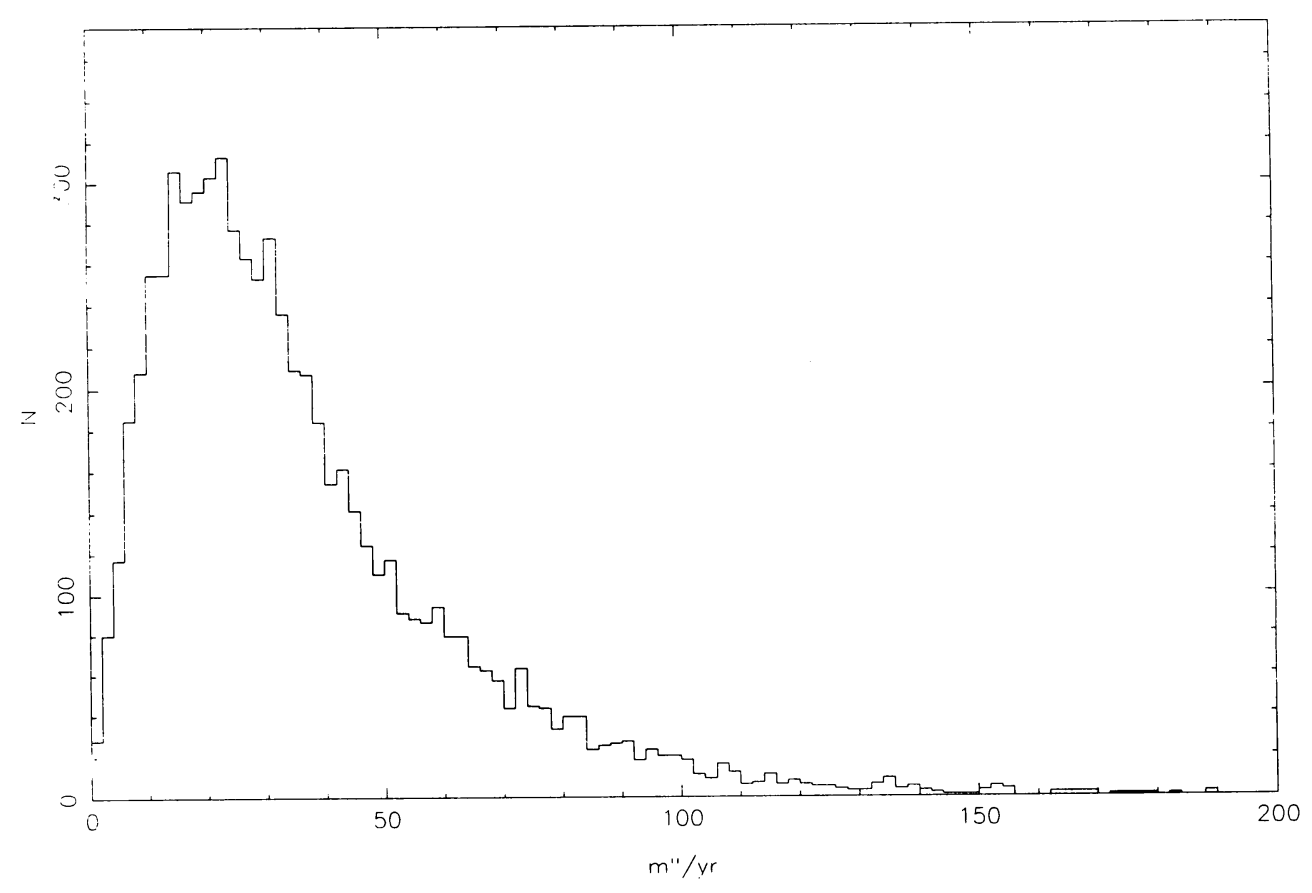

Figure 2.

Given the unique combination of great depth and large field, it was relatively straightforward to look for stars with a large R-I colour. Some 40 candidates were found with R-I $>3$, the colour of the reddest dwarf stars currently known. Several stars were found with $R-I \approx 4$, one of which is illustrated in Fig. 3. The top plot is from the red stack and shows a faint image in the middle. The same object appears much brighter relative to nearby stars in the I stack shown below. A spectrum has been obtained for this star which shows the coolest dwarf so far discovered. Proper motions were measured for the sample from I plates where the images were brightest, which implied that sample measures were up to 3 magnitudes less luminous than any known dwarf. The brightest stars in the sample had also been detected from single plate surveys, and comparisons showed excellent agreement between magnitudes and proper motions from the two samples.

Another early project was an extension of a large scale survey for variable quasars. The survey was based on single plates and was effective at finding quasars up to redshift of about 3.2. At higher redshifts quasars become very faint and stacked data provide a means of searching to greater depths. The variability measures were achieved as for proper motions, by stacking the data in small groups. Several high redshift quasars have already been found from their variability. The light curves of two found from the red stack are shown in Fig. 4. The photometric accuracy is quite sufficient to detect objects with amplitudes around half a magnitude, which is typical for quasars.

Some dozen projects are now underway using the stacked data, covering a wide variety of 
$77752 \quad 202049$

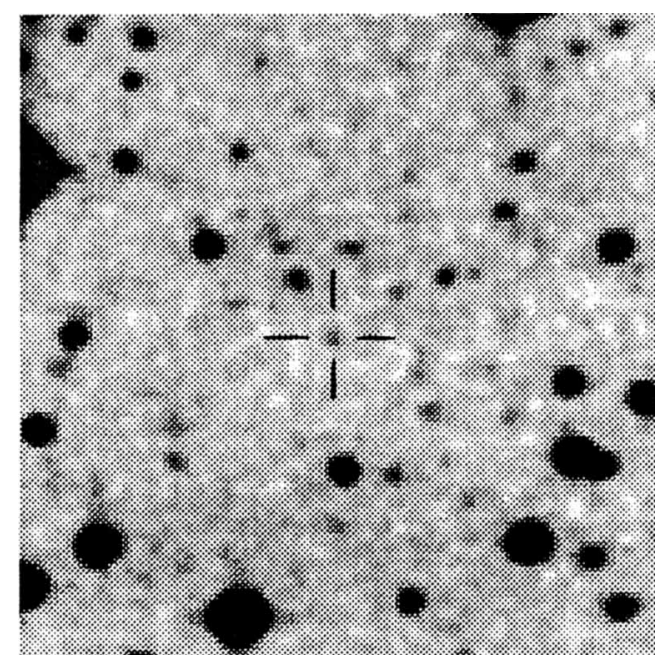

$77752 \quad 202049$

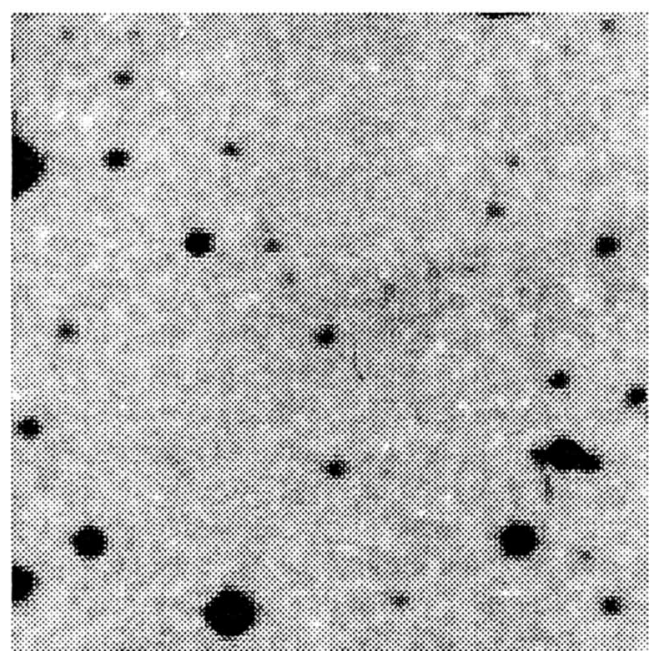

Figure 3. 

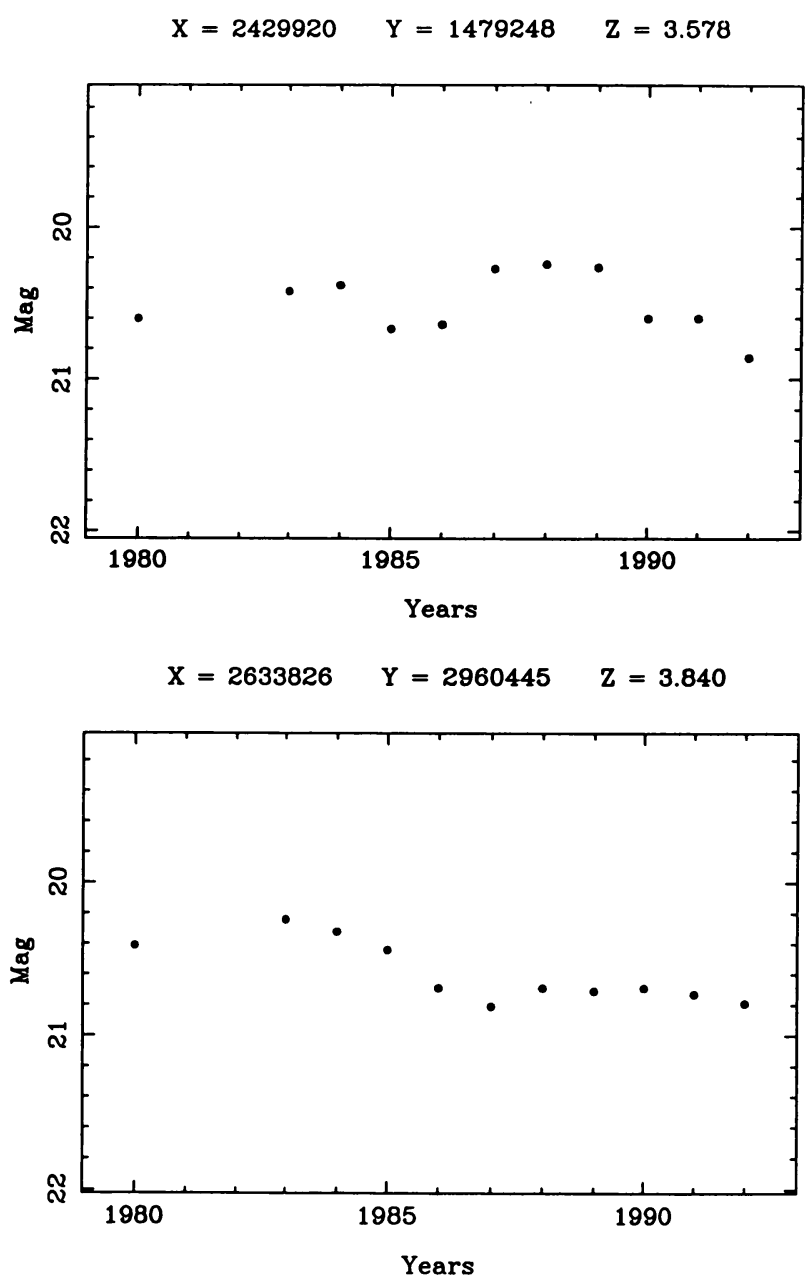

Figure 4.

topics. The next few years should provide many exciting results as techniques for using the stacked data are developed and the new projects come to fruition. 